\title{
Nonclinical Study and Applicability of the Absorbed Dose Conversion Method With a Single Biodistribution Measurement for Targeted Alpha- Nuclide Therapy
}

\section{Tetsuya Sakashita ( $\nabla$ sakashita.tetsuya@qst.go.jp )}

National Institutes for Quantum and Radiological Science and Technology https://orcid.org/00000002-3357-8373

\section{Shojiro Matsumoto}

National Institutes for Quantum and Radiological Science and Technology

\section{Shigeki Watanabe}

National Institutes for Quantum and Radiological Science and Technology Hirofumi Hanaoka

Gunma University Graduate School of Medicine

\section{Yasuhiro Ohshima}

National Institutes for Quantum and Radiological Science and Technology

\section{Yoko Ikoma}

National Institutes for Quantum and Radiological Science and Technology

\section{Naoyuki Ukon}

Fukushima Medical University

Ichiro Sasaki

National Institutes for Quantum and Radiological Science and Technology

\section{Tatsuya Higashi}

National Institutes for Quantum and Radiological Science and Technology

\section{Tetsuya Higuchi}

Gunma University Graduate School of Medicine

Yoshito Tsushima

Gunma University Graduate School of Medicine

Noriko S. Ishioka

National Institutes for Quantum and Radiological Science and Technology

\section{Original research}

Keywords: Targeted alpha-nuclide therapy, Dose conversion, Biodistribution, Pharmacokinetics, RAP 
Posted Date: June 7th, 2021

DOl: https://doi.org/10.21203/rs.3.rs-573658/v1

License: (c) (i) This work is licensed under a Creative Commons Attribution 4.0 International License. Read Full License

Version of Record: A version of this preprint was published at EJNMMI Physics on December 1st, 2021. See the published version at https://doi.org/10.1186/s40658-021-00425-z. 
1

2

3

4

5

6

7 Tetsuya Sakashita ${ }^{1, *}$, Shojiro Matsumoto ${ }^{1}$, Shigeki Watanabe ${ }^{1}$, Hirofumi Hanaoka ${ }^{2}$,

8 Yasuhiro Ohshima ${ }^{1}$, Yoko Ikoma ${ }^{3}$, Naoyuki Ukon ${ }^{4}$, Ichiro Sasaki ${ }^{1}$, Tatsuya Higashi ${ }^{3}$,

9 Tetsuya Higuchi ${ }^{5}$, Yoshito Tsushima ${ }^{5}$, Noriko S. Ishioka ${ }^{1}$

Original research articles

\section{Nonclinical study and applicability of the absorbed dose conversion} method with a single biodistribution measurement for targeted alphanuclide therapy

6

$11{ }^{1}$ Quantum Beam Science Research Directorate, National Institutes for Quantum and

12 Radiological Science and Technology, 1233 Watanuki-machi, Takasaki 370-1292, Japan

$13{ }^{2}$ Department of Bioimaging Information Analysis, Gunma University Graduate School of

14 Medicine, 3-39-22 Showa, Maebashi 371-8511, Japan

$15{ }^{3}$ Department of Molecular Imaging and Theranostics, National Institutes for Quantum and

16 Radiological Science and Technology, 4-9-1 Anagawa, Inage-ku, Chiba 263-8555, Japan

$17{ }^{4}$ Advanced Clinical Research Center, Fukushima Medical University, 1 Hikariga-oka, 18 Fukushima 960-1295, Japan 
$1{ }^{5}$ Department of Diagnostic Radiology and Nuclear Medicine, Gunma University

2 Graduate School of Medicine, 3-39-22 Showa, Maebashi 371-8511, Japan

3

4 *Corresponding author: Dr. Tetsuya Sakashita, Quantum Beam Science Research

5 Directorate, National Institutes for Quantum and Radiological Science and Technology,

61233 Watanuki-machi, Takasaki 370-1292, Japan. Tel.: +81-27-346-9103; Fax: +81-27-

$7 \quad 346-9688$. E-mail: sakashita.tetsuya@qst.go.jp

8 
1 Abstract

2 Background: We recently reported a new absorbed dose conversion method, RAP(RAtio

3 of Pharmacokinetics), for ${ }^{211}$ At-meta-astatobenzylguanidine ( ${ }^{211}$ At-MABG) using a

4 single biodistribution measurement $(\% \mathrm{ID} / \mathrm{g})$. However, there were some mathematical

5 ambiguities in determing the optimal timing of a single measurement of $\% \mathrm{ID} / \mathrm{g}$. Thus, we

6 aimed to mathematically reconstruct the RAP method and to examine the optimal timing

7 of a single measurement.

8 Results: We derived a new formalism of the RAP dose conversion method at time $t$ and

9 investigated the new formalism's performance using a representative RAP coefficient

10 with radioactive-decay weighting. Dose conversions by representative RAP coefficients

11 predicted the true $\left[{ }^{211} \mathrm{At}\right] \mathrm{MABG}$ absorbed doses with an error of $10 \%$ or less. The inverses

12 of the representative RAP coefficients plotted at $4 \mathrm{~h}$ post-injection, which was the optimal

13 timing reported in the previous work, were very close to the new inverses of the RAP

14 coefficients $4 \mathrm{~h}$ post-injection. Next, we acquired a formula to determine the optimal

15 timing of a single measurement of $\% \mathrm{ID} / \mathrm{g}$, assuming the one-compartment model for

16 biological clearance. The behavior of the optimal timing was analyzed by radiolabeled

17 compounds with physical half-lives of $7.2 \mathrm{~h}$ and $10 \mathrm{~d}$ on various biological clearance half-

18 lives. Behavior maps of optimal timing showed a tendency to converge to a constant value 
1 as the biological clearance half-life of a target increased. The areas of optimal timing for

2 both compounds within a $5 \%$ or $10 \%$ prediction error were distributed around the optimal

3 timing when the biological clearance half-life of a target was equal to that of the reference.

4 Finally, an example of RAP dose conversion was demonstrated for [ $\left.{ }^{211} \mathrm{At}\right] \mathrm{MABG}$.

5 Conclusions: The RAP dose conversion method renovated by the new formalism was

6 able to estimate the $\left[{ }^{211} \mathrm{At}\right] \mathrm{MABG}$ absorbed dose using a similar pharmacokinetics, such

7 as $\left[{ }^{131} \mathrm{I}\right] \mathrm{MIBG}$. The present formalism revealed optimizing imaging time points on

8 absorbed dose conversion between two radiopharmaceuticals. Further analysis and

9 clinical data will be needed to elucidate the validity of a behavior map of the optimal

10 timing of a single measurement for targeted alpha-nuclide therapy.

12 Keywords: Targeted alpha-nuclide therapy, Dose conversion, Biodistribution,

13 Pharmacokinetics, RAP. 
1

$2{ }^{211}$ At-meta-astatobenzylguanidine $\left({ }^{211} \mathrm{At}-\mathrm{MABG}\right)$ is a potential nuclear medicine for 3

6 undergo ${ }^{211}$ At-MABG treatment for remission. In ${ }^{131}$ I-MIBG therapy, diagnostic imaging

7 by ${ }^{131}$ I-scintigraphy and/or SPECT during ${ }^{131}$ I-MIBG treatment would be performed occasionally as part of follow-up care, because such imaging is of clinical prognostic

9 value. Predicting the organ or tumor tissue absorbed dose of such patients in ${ }^{211}$ At-MABG

10 treatment using ${ }^{131}$ I-MIBG image data would be useful for planning ${ }^{211}$ At-MABG therapy.

11 Therefore, it is important to develop methods for estimating ${ }^{211}$ At-MABG doses using ${ }^{131}$ I-MIBG biodistribution data. At present, one of issues in systemic radiopharmaceutical therapy or TAT is optimizing imaging time points [2]. The previous work of Madsen et al. [3] revealed a single-time to estimate the total integrated activity and absorbed dose to within $10 \%$ accuracy. Recently, we reported a novel dose conversion method, RAP (RAtio of

17 Pharmacokinetics), using biodistribution data, namely the percent injected dose/g $18(\% \mathrm{ID} / \mathrm{g})[4]$. In that study, we extended optimizing imaging time points on absorbed dose 
1 conversion between two radiopharmaceuticals, and demonstrated that the RAP dose

2 conversion method could estimate ${ }^{211}$ At-MABG absorbed doses from a single

3 measurement of $\% \mathrm{ID} / \mathrm{g}$ and the pharmacokinetics of ${ }^{131} \mathrm{I}-\mathrm{MIBG}$ in a $24 \mathrm{~h}$ evaluation

4 period. However, at that time we were unable to present a sufficient mathematical

5 approach for the timing of a single measurement of $\% \mathrm{ID} / \mathrm{g}$. Moreover, only proportional

6 relations formed the mathematical basis of the RAP method, resulting in some

7 mathematical ambiguities. Therefore, we aimed to describe the RAP method

8 mathematically without proportional relations and to examine the timing of a single

9 measurement of $\% \mathrm{ID} / \mathrm{g}$.

10 In the present paper, we proposed a novel formula to estimate the optimal timing

11 of a single measurement of $\% \mathrm{ID} / \mathrm{g}$ and analyzed the behavior of the optimal timing

12 depending on the biological pharmacokinetic parameters. Also, we used two other sets

13 with a short evaluation period (6 h): ${ }^{77} \mathrm{Br}-$ meta-bromobenzylguanidine $\left({ }^{77} \mathrm{Br}-\mathrm{MBBG}\right)$ and

$14{ }^{125}$ I-meta-iodobenzylguanidine $\left({ }^{125} \mathrm{I}-\mathrm{MIBG}\right)$ to examine the performance of the RAP

15 conversion. Moreover, we present an example of RAP dose conversion using a behavior

16 map of the optimal timing of a single measurement of $\% \mathrm{ID} / \mathrm{g}$. 
1 Simulation datasets

2 On time-series of biodistribution profiles in our previous Monte Carlo simulation work

3 on ${ }^{211}$ At-MABG, ${ }^{131} \mathrm{I}-\mathrm{MIBG},{ }^{77} \mathrm{Br}-\mathrm{MBBG}$, and ${ }^{125} \mathrm{I}-\mathrm{MIBG}$, we used a total of 8,000

4 simulation (virtual experiment) datasets [4]. In the present study, we applied a median of

5200 simulation datasets at each organ or tumor tissue. The simulation was carried out

6 based on the biodistribution profiles of three reports at several time points of seven organs

7 (heart, liver, kidney, intestine, blood, adrenals, stomach) and tumor tissue [3, 4, 5]. One

8 of these reports, by Vaidyanathan et al., reported the biodistributions of ${ }^{211}$ At-MABG and

$9{ }^{131} \mathrm{I}-\mathrm{MIBG}$ in nude mice with SK-N-SH human neuroblastoma xenografts [5]. In the

10 second, by Ohshima et al. [6], a rat PC12 pheochromocytoma model was used to examine

11 the antitumor effects of ${ }^{211}$ At-MABG. The third report, by Watanabe et al. [7], analyzed

12 the biodistributions of ${ }^{77} \mathrm{Br}-\mathrm{MBBG}$ and ${ }^{125} \mathrm{I}-\mathrm{MIBG}$ using PC12 xenografts. We have

13 labeled the simulated biodistribution datasets created from these previous reports as

14 [ $\left.{ }^{211} \mathrm{At}\right] \mathrm{MABG}[5],\left[{ }^{131} \mathrm{I}\right] \mathrm{MIBG}[5],\left[{ }^{211} \mathrm{At}\right] \mathrm{MABG}$ [6], $\left[{ }^{77} \mathrm{Br}\right] \mathrm{MBBG}$ [7], and $\left[{ }^{125} \mathrm{I}\right] \mathrm{MIBG}$

15 [7], respectively. Their experimental conditions are shown in Table 1.

17 New formalism of RAP method at time $t$

18 In our previous work, we could not present a sufficient mathematical approach to the 
1 timing of a single measurement. The mathematical basis of the RAP method was the

2 proportional relation as follows:

3

7 and $\frac{1}{\frac{\% \mathrm{ID} / \mathrm{g}\left({ }^{131} \mathrm{I}\right)}{\% \mathrm{ID} / \mathrm{g}\left({ }^{211} A t\right)}}$ is the RAP coefficient defined in the previous work [4]. There were

some mathematical ambiguities.

Here, we show a new derivation method for the RAP formula based on the activity concentration $(\mathrm{kBq} / \mathrm{g})$ of an organ or tumor tissue, $C(t)$. In the previous work, $C$ $(t)$ was expressed using the two-biological-compartments model for normal organs except adrenals:

$$
C(t)=C_{0} \exp \left(-\frac{\ln (2)}{T_{p}} t\right)\left\{f \exp \left(-\frac{\ln (2)}{T_{b 1}} t\right)+(1-f) \exp \left(-\frac{\ln (2)}{T_{b 2}} t\right)\right\}
$$

where $C(t)$ is the activity concentration for the normal organ at time (sec) $t$ post-injection, $C_{0}$ is the initial activity concentration, $T_{p}$ is the physical HL time (sec), and $f$ and $(1-f)$ are the fractions of the two biological compartments on clearance. $T_{b 1}$ and $T_{b 2}$ are the corresponding HL times (sec) for fast and slow biological clearances, respectively. Or, in the case of adrenals and tumor tissue, the following one-compartment equation was used:

$$
C(t)=C_{0}\left(1-\exp \left(-\frac{\ln (2)}{T_{u p}} t\right)\right) \exp \left(-\frac{\ln (2)}{T_{p}} t\right) \exp \left(-\frac{\ln (2)}{T_{b 1}} t\right)
$$


where $T_{u p}$ is the HL time of uptake (sec). injection:

6

$$
C(t)=I D C_{0} \exp \left(-\frac{\ln (2)}{T_{p}} t\right)(\% \mathrm{ID} / \mathrm{g})(t)
$$

7 where, in the previous simulation work, we assumed an injection with $100 \mathrm{kBq}$ of ${ }^{211}$ AtMABG in $100 \mu \mathrm{L}$ of PBS into a tail vein and around $1 \mathrm{MBq}$ as the total activity of a mouse. Next, we applied this equation to two radiolabeled compounds, $A_{1}$ and $A_{2}$.

$$
\begin{aligned}
& C_{-} A_{1}(t)=I D C_{0 \_} A_{1} \exp \left(-\frac{\ln (2)}{T_{p A_{1}}} t\right)(\% \mathrm{ID} / \mathrm{g})_{A_{1}}(t) \text { and } \\
& C_{-} A_{2}(t)=I D C_{0 \_} A_{2} \exp \left(-\frac{\ln (2)}{T_{p A_{2}}} t\right)(\% \mathrm{ID} / \mathrm{g})_{A_{2}}(t) .
\end{aligned}
$$

By dividing and transforming both sides of the (4-1) and (4-2) equations, we described the following new derivative relation for the RAP formalism:

$$
C_{-} A_{1}(t)=C_{-} A_{2}(t) \frac{I D C_{0} A_{1}}{I D C_{0-} A_{2}} \frac{\exp \left(-\frac{\ln (2)}{T_{p A_{1}}} t\right)}{\exp \left(-\frac{\ln (2)}{T p A_{2}} t\right)} \frac{1}{\frac{(\% \mathrm{ID} / \mathrm{g}) A_{2}(t)}{(\% \mathrm{ID} / \mathrm{g}) A_{1}(t)}} .
$$

15 Here, $\frac{1}{\frac{(\% \mathrm{ID} / \mathrm{g}) A_{2}(t)}{(\% \mathrm{ID} / \mathrm{g})_{A_{1}}(t)}}$ is the RAP coefficient at $t$. We assumed $I D C_{0}$ to $1 \mathrm{MBq} / \mathrm{ml}$ for both radiolabeled compounds $A_{1}$ and $A_{2}$ and set $I D C_{0} A_{1} / I D C_{0} A_{2}$ to 1 . If the injected dose concentration is different, we recommend normalizing it to $1 \mathrm{MBq} / \mathrm{ml}$ and multiplying the required concentration $\mathrm{x}$ times $(\mathrm{MBq} / \mathrm{ml})$, to the calculated result. To calculate a time 
1 integration activity concentration (TIAC) (Bq-h/g) of radiolabeled compounds $A_{1}$ and $A_{2}$,

2 we numerically integrated equation (5) with a $1 \mathrm{~h}$ interval.

3 The absorbed radiation dose (Gy), $D$, for normal or tumor tissue was calculated

4 according to the following modified MIRD formalism:

5

$$
D=1000 \cdot T I A C \cdot E \cdot F \cdot P,
$$

6 where the energy emitted by ${ }^{211} \mathrm{At}, E$, is assumed to be solely from the alpha

7 disintegrations, corresponding to $6.9 \mathrm{MeV} / \mathrm{Bq}-\mathrm{s}$ [8]. The absorbed fraction, $F$, is set to

81 , since it is assumed that all energy emitted by ${ }^{211}$ At is absorbed by the source tissue or

9 organ. $P$ is the coefficient for converting from $\mathrm{g}$ to $\mathrm{kg}, 1000$. Finally, the absorbed dose

$10(\mathrm{~J} / \mathrm{kg}=\mathrm{Gy})$ was calculated using the relation of $1.60210^{-13}(\mathrm{~J} / \mathrm{MeV})$.

12 Framework for practical use of the RAP dose conversion

13 In the present work, we set radiolabeled compound $\mathrm{A}_{2}$, which has a well-known biological

14 kinetics, as a reference, and radiolabeled compound $A_{1}$ as a target with unknown

15 biological kinetics. Our goal is to convert from the absorbed dose of $\mathrm{A}_{2}$ to that of $\mathrm{A}_{1}$.

16 Integrating equation (5) leads to a TIAC $(\mathrm{Bq}-\mathrm{h} / \mathrm{g})$ of $\mathrm{A}_{1}$, but, in the case of unknown

17 biological kinetics of $A_{1}$, integration would be difficult. On the other hand, the physical

18 part of equation (5), that is, equation (5) except for the RAP coefficient, could be easily 
1 integrated if the pharmacokinetics of $A_{2}$ is well known. Here, it should be noted that a

2 single measurement of $\% \mathrm{ID} / \mathrm{g}$ has been used to demonstrate successful RAP dose

3 conversion [4]. In short, we needed to work on simplifying the integration of the RAP

4 coefficients, and used simulation datasets from the previous work to achieve that. the physical part of equation (5) except for the RAP coefficient. We also calculated the mean value of the target's radioactive-decay weighted RAP coefficients during the

8 evaluation period, representative RAP coefficient. TIACs were obtained by multiplying

9 the integral value of the physical part by the representative RAP coefficient. Finally,

10 absorbed doses were estimated using equation (6). We labeled the absorbed dose based

11 on the TIACs that were considered the physical part of the integration as "with HL", and that considered TIACs multiplied by the representative RAP coefficient as "with HL + 13 RAP”.

In the next attempt, the generalization of the RAP method, we acquired the formula for the optimal timing of a single measurement of $\% \mathrm{ID} / \mathrm{g}$, assuming the onecompartment model for biological clearance. The TIAC of $\mathrm{A}_{1}, T I A C_{-} A_{1}$, and that of $\mathrm{A}_{2}$,

17 TIAC_A $A_{2}$, with HL were expressed by the following equations, respectively: 
1

2

3

4

$$
T I A C_{-} A_{1}=\frac{A_{0-} A_{1}}{-\ln 2\left(\frac{1}{T_{p A_{1}}}+\frac{1}{T_{b A_{1}}}\right)}\left[\exp \left(-\ln 2\left(\frac{1}{T_{p A_{1}}}+\frac{1}{T_{b A_{1}}}\right) t\right)\right]_{t_{0}}^{t_{1}}
$$

$$
\text { TIAC_A } A_{2} \text { with } H L=\frac{A_{0 \_} A_{2}}{-\ln 2\left(\frac{1}{T_{p A_{1}}}+\frac{1}{T_{b A_{2}}}\right)}\left[\exp \left(-\ln 2\left(\frac{1}{T_{p A_{1}}}+\frac{1}{T_{b A_{2}}}\right) t\right)\right]_{t_{0}}^{t_{1}},
$$

where $A_{0} A_{1}$ and $A_{0} A_{1}$ are the initial activity concentrations, and $T_{b A 1}$ and $T_{b A 2}$ are HLs for biological clearances of radiolabeled compounds $A_{1}$ and $A_{2}$, respectively. $t_{0}$ and $t_{1}$ are the start and end times of the evaluation period. Here, the optimal timing of a single measurement of $\% \mathrm{ID} / \mathrm{g}$ should satisfy the following relation because of equations (1):

$$
\text { RAP coefficient }=\frac{T I A C_{-} A_{1}}{T I A C_{-} A_{2} \text { with } H L} \text {. }
$$

Then, the optimal timing, Opt_t, was solved as follows:

$0 p t_{-} t=\frac{1}{-\ln 2\left(\frac{1}{T_{b A_{1}}}-\frac{1}{T_{b A_{2}}}\right)} \ln \frac{\frac{1}{\frac{1}{T_{p A_{1}}}+\frac{1}{T_{b A_{1}}}}\left[\exp \left(-\ln 2\left(\frac{1}{T_{p A_{1}}}+\frac{1}{T_{b A_{1}}}\right) t\right)\right]_{t_{0}}^{t_{1}}}{\frac{1}{\frac{1}{T_{p A_{1}}}+\frac{1}{T_{b A_{2}}}}\left[\exp \left(-\ln 2\left(\frac{1}{T_{p A_{1}}}+\frac{1}{T_{b A_{2}}}\right) t\right)\right]_{t_{0}}^{t_{1}}}$ of $\% \mathrm{ID} / \mathrm{g}$.

\section{Results}

\section{Analysis of the RAP coefficients}


1 For the analysis of the RAP coefficients, we prepared the converted absorbed doses of

$2 \quad\left[{ }^{131} \mathrm{I}\right] \mathrm{MIBG}[5],\left[{ }^{211} \mathrm{At}\right] \mathrm{MABG}[6],\left[{ }^{125} \mathrm{I}\right] \mathrm{MIBG}$ [7], and $\left[{ }^{77} \mathrm{Br}\right] \mathrm{MBBG}$ [7] as references

3 and the true absorbed dose of $\left[{ }^{211} \mathrm{At}\right] \mathrm{MABG}[5]$ as a target, using the simulation datasets

4 of the previous work [4]. Here, for example, the converted absorbed dose of [ $\left.{ }^{131} \mathrm{I}\right] \mathrm{MIBG}$

5 [5] indicates the absorbed dose of an organ or tumor tissue when alpha rays derived from

$6 \quad{ }^{211}$ At were emitted by the number of radioactive decays of ${ }^{131}$ I. To investigate the relation

7 between the RAP coefficient and the optimal timing of a single measurement of $\% \mathrm{ID} / \mathrm{g}$,

8 we produced a new derivative relation for RAP formalism, equation (5). In our first

9 attempt at equation (5), we employed radioactive-decay weighting for the RAP

10 coefficients at $t$ and calculated the representative RAP coefficient for the evaluation

11 period. We multiplied the representative RAP coefficient by the physical HL-corrected

12 TIAC of a reference. Then, we calculated absorbed doses based on the physical HL-

13 corrected TIAC, that is, "with HL", and the physical HL-corrected and RAP-converted

14 TIAC, which we labeled "with HL + RAP".

Figure 1 shows the converted absorbed doses of the references, the "with HL"-

16 corrected ones, the "with HL + RAP" ones, and the true absorbed dose of the

$17 \quad\left[{ }^{211} \mathrm{At}\right] \mathrm{MABG}[5]$ target. Most “with HL + RAP” converted absorbed doses were closer

18 to the true absorbed dose of $\left[{ }^{211} \mathrm{At}\right] \mathrm{MABG}$ than the "with HL" ones. Average percent 
1 differences between converted and true absorbed [ $\left.{ }^{211} \mathrm{At}\right] \mathrm{MABG} \_1996[5]$ doses were -

$223 \%$ in "with HL" of $\left[{ }^{131}\right.$ I]MIBG [5] and 7\% in "with HL + RAP". Similarly, those of

$3 \quad\left[{ }^{211} \mathrm{At}\right] \mathrm{MABG}[6]$ were $112 \%$ and $-7 \%$, those of $\left[{ }^{125} \mathrm{I}\right] \mathrm{MIBG}[7]$ were $53 \%$ and $-2 \%$, and

4 those of $\left[{ }^{77} \mathrm{Br}\right] \mathrm{MBBG}[7]$ were $74 \%$ and $-2 \%$ (Table 2). These results showed that the

5 dose conversions by the representative RAP coefficients were able to predict the true

6 value with an error of $10 \%$ or less.

To examine the ratio in $\% \mathrm{ID} / \mathrm{g}$ of a reference to a target, we plotted the inverse

8 of the RAP coefficient (Figure 2). The inverses of the RAP coefficients at $t$ changed over

9 time in the seven organs and the tumor tissue. The inverses of the representative RAP

10 coefficients were plotted at $4 \mathrm{~h}$ post-injection, which was the optimal timing reported in

11 the previous work [4]. As a result, we found that the inverse values of RAP coefficients 4

$12 \mathrm{~h}$ post-injection and the representative RAP coefficient were very close to each other.

13 Taken together, these results suggest that the new RAP coefficients at $4 \mathrm{~h}$ post-injection

14 corresponded to the RAP coefficients in the previous work.

16 Behavior of optimal timing of a single biodistribution measurement

17 The representative RAP coefficient needs to be based on the detailed kinetics of

18 radiolabeled compound $\mathrm{A}_{1}$ as a target, but we could estimate the ${ }^{211} \mathrm{At}-\mathrm{MABG}$ absorbed 
1 dose from a single biodistribution measurement. We should return to the origin of the

2 RAP dose conversion, which is equation (8). Therefore, an optimal timing needs to satisfy

3 the relation in equation (8). Assuming the one-compartment model for biological

4 clearance, we calculated TIACs of $A_{1}$ and $A_{2}$ analytically, and the optimal timing was

5 solved by equation (9). Interestingly, equation (9) did not include the physical HL time of

6 radiolabeled compound $\mathrm{A}_{2}$. Using this equation, we investigated the behavior of the

$7 \quad$ optimal timing of a single measurement of $\% \mathrm{ID} / \mathrm{g}$.

8 The behavior of an optimal timing on ${ }^{211}$ At-labeled $A_{1}$ was estimated

9 depending on the HLs of the biological clearance of a target, $T_{b A 1}$, from 0 to $100 \mathrm{~h}$ and the

10 HLs of the biological clearance of a reference, $T_{b A 2}$, of 5, 10, 15, 20, 25, and $50 \mathrm{~h}$ (Figure

11 3). Here, we set the end time of the evaluation period to $72 \mathrm{~h}$, which was 10 times the HL

12 of ${ }^{211}$ At. In addition, we plotted $0.9,0.95,1.05$, and 1.1 times TIACs of $A_{1}$ in order to

13 understand the area of optimal timing where the absorbed dose could be estimated within

14 a $5 \%$ or $10 \%$ prediction error. As shown in figure 3 , the optimal timing tended to converge

15 to a constant value as $T_{b A 1}$ increased. Interestingly, all optimal timing values were

16 acceptable when $T_{b A 1}$ was equal to $T_{b A 2}$. The area of an optimal timing within a $5 \%$ or

$17 \quad 10 \%$ prediction error was distributed around the intersection where the curve of optimal

18 timing agreed with the line where $T_{b A 1}$ was equal to $T_{b A 2}$. 
1

$210 \mathrm{~d}$, e.g., ${ }^{225} \mathrm{Ac}$, depending on $T_{b A 1}$ from 0 to $250 \mathrm{~h}$ and $T_{b A 2}$ of $25,50,100$, and $200 \mathrm{~h}$.

3 Here, we set the end time of the evaluation period to 2,400 $\mathrm{h}$. The optimal timing also

Figure 4 shows the behavior of an optimal timing on $\mathrm{A}_{1}$ with a physical HL of tended to converge to a constant value as $T_{b A 1}$ increased, and the convergence value was larger than that of ${ }^{211}$ At. The area of optimal timing within a $5 \%$ or $10 \%$ prediction error was also distributed around the intersection where the curve of optimal timing agreed with the line where $T_{b A 1}$ was equal to $T_{b A 2}$. In addition, an enlarged view of the behavior map at $25 \mathrm{~h} T_{b A 2}$ shows that the behavior was almost the same as that for ${ }^{211}$ At except for a different convergence value (Figure 5a). Moreover, short HLs of biological clearance in $T_{b A 1}$ and $T_{b A 2}$ displayed short optimal timing (Figure $5 \mathrm{~b}$ ). These results suggest that the area of optimal timing depended on both $T_{b A 1}$ and $T_{b A 2}$, and that the convergence value of the optimal timing was controlled by the physical HL time of radiolabeled compound $\mathrm{A}_{1}$.

4 Example of RAP dose conversion using optimal timing of a single biodistribution measurement.

Analysis of RAP coefficients and of a behavior map of the optimal timing of a single measurement of $\% \mathrm{ID} / \mathrm{g}$ confirmed the practical applicationof the RAP method. Here, we present a case of the heart in $\left[{ }^{131} \mathrm{I}\right] \mathrm{MIBG}[5]$ as a reference and $\left[{ }^{211} \mathrm{At}\right] \mathrm{MABG}[5]$ as a 
1 target, using the simulation datasets from the previous work [4].

3 the physical half-life of ${ }^{131}$ I and fitted by the linear function, which was " $a t+b$ " (Figure

4 6a). The fitted function was $-0.1006 \mathrm{t}+4.7768\left(\mathrm{R}^{2}=0.926\right)$. From this relation, we

5 estimated that the HL of the biological clearance of a reference, $T_{b A 2}$, was $6.89 \mathrm{~h}$. Next,

6 we drew the behavior map of the optimal timing on $\mathrm{A}_{1}$ labeled with ${ }^{211}$ At and with $T_{b A 2}$

7 of $6.9 \mathrm{~h}$ (Figure $6 \mathrm{~b}$ ). From the behavior map, we decided that $5 \mathrm{~h}$ of optimal timing,

8 assuming the HL of the biological clearance of a target, $T_{b A 1}$, was close to that of $T_{b A 2}$. We

9 calculated the inverse of the RAP coefficient from the values of $\% \mathrm{ID} / \mathrm{g}$ in simulation

10 datasets of radiolabeled compound $\mathrm{A}_{1}$ and radiolabeled compound $\mathrm{A}_{2}$, which was 0.76 .

11 Finally, we estimated an absorbed dose of 3.3 Gy of $\left[{ }^{211} \mathrm{At}\right] \mathrm{MABG}$ [5], by multiplying

12 the RAP coefficient by that of the physical HL-corrected absorbed dose, 2.7 Gy, which is

13 a numerical integration of the physical part of equation (5) (Figure 6c). The difference

14 between the RAP-converted absorbed dose and a true $\left[{ }^{211}\right.$ At]MABG_1996[5] absorbed dose was $7 \%$, which was superior to the $10 \%$ prediction error obtained by the representative RAP coefficient in Table 2. This result suggests that the present RAP dose conversion method is practical enough to use. 


\section{Discussion}

2 In this study, we focused on the mathematical ambiguities we encountered in our

3 derivation of RAP coefficients in our previous work. We aimed to describe the RAP

4 method mathematically without proportional relations and to examine the timing of a

5 single biodistribution measurement $(\% \mathrm{ID} / \mathrm{g})$. Here, we proposed a novel formula to

6 estimate the optimal timing of a single measurement of $\% \mathrm{ID} / \mathrm{g}$. Analysis of the behavior

7 on the optimal timing revealed that the physical HL time of a target radiolabeled

8 compound depended on the biological clearances of both the target and reference

9 radiolabeled compounds. Finally, we presented an example of a practical use of RAP dose

10 conversion.

A behavior map of an optimal timing on a single measurement of $\% \mathrm{ID} / \mathrm{g}$ might

12 lead to several important strategies for RAP dose conversion. The first strategy involves

13 a selection of a long biological clearance of a reference against a target with short and

14 long physical HLs. If we could select a long biological clearance of a reference, we would

15 have a broad window for optimal timing of a single biodistribution measurement (Figures

163 and 4). For example, in the case of a target with $10 \mathrm{~d}$ physical HL, over $25 \mathrm{~h}$ of the

17 biological clearance HLs of a target and a reference could make an almost $1 \mathrm{~h}$ window

18 for optimal timing (figure 5a). The second is the operation of the short biological 
1 clearance HLs of a target and of a reference as shown in figure 5b. Even with a long

2 physical HL, e.g., ${ }^{225}$ Ac, it might be possible to obtain the RAP coefficient within a

3 feasible and short time by this operation, although it would be limited to a case of

4 appropriate drug delivery. For example, the HLs of the biological clearance of nuclear

5 medicine using the prostate-specific membrane antigen (PSMA) in animal studies were

6 around $1 \mathrm{~h}$ [9]. A behavior map of an optimal timing might make it possible to present an

7 appropriate imaging plan with a single measurement biodistribution for nuclear medicines

8 that are difficult to measure multiple times.

period. If a one-compartment assumption for biological clearance holds for infinite time,

11 the optimal timing equation could be transformed as follows:

$$
O p t_{-} t=\frac{1}{-\ln 2\left(\frac{1}{T_{b A_{1}}}-\frac{1}{T_{b A_{2}}}\right)} \ln \frac{\frac{1}{T_{p A_{1}}}+\frac{1}{T_{b A_{2}}}}{\frac{1}{T_{p A_{1}}}+\frac{1}{T_{b A_{1}}}}
$$

where $O p t_{-} t$ is an optimal timing, $T_{p A l}$ is the physical HL of a target, $T_{b A 1}$ is the biological clearance HL of a target, and $T_{b A 2}$ is the biological clearance HL of a reference. However, verifiable data are rarely available for infinite time. In this paper, we proposed equation (9) of the optimal timing with a limited evaluation period, so that we could apply it even when the activity concentration data were available for only a short evaluation period. In 
1 timing formulated by equation (9) has limited application because of an assumption of

2 the one-compartment model for biological clearance. This one-compartment assumption

3 does not hold in the early stages of the evaluation period, when the uptake of radiolabeled

4 compounds continues for a long time, as in equation (3). Therefore, in this case it is

5 necessary to adjust the start time of the evaluation. This is a future issue.

6 We should note that RAP dose conversion using a behavior map did not require

7 difficult formulas and techniques. We would need only the physical HL correction, linear

8 fitting, the calculations of equation (9), and a numerical integration such as a trapezoidal

9 quadrature. For example, most researchers would use absorbed dose estimation software,

10 e.g., Organ Level INternal Dose Assessment/EXponential Modeling (OLINDA/EXM)

11 (Vanderbilt University, Nashville, TN, USA) [10]. Using these programs, users estimate

12 the time activity curve (TAC) of a radionuclide-labeled compound fitted by the

13 exponential functions. The value per unit weight of the TAC corresponds to $C(t)$ in the

14 present equation (4) and input for the example as shown in figure 6, indicating the

15 feasibility of RAP dose conversion. OLINDA/EXM users could also use RAP dose

16 conversion.

17 Unfortunately, clinical RAP coefficient information does not exist, and

18 consideration of the RAP coefficient in animal biodistribution studies is currently limited. 
1 Also, an approximation of the one-compartment model applied to a behavior map of an

2 optimal timing might create limitations in use. However, even in the biological clearance

3 of $\left[{ }^{131} \mathrm{I}\right] \mathrm{MIBG}$ [5] with a two-compartment phase [11], the RAP dose conversion

4 displayed superior prediction error of less than $10 \%$. The approach presented in a behavior

5 map of an optimal timing on a single biodistribution measurement might provide useful

6 information for the treatment planning of ${ }^{211}$ At-MABG therapy or TAT. Taken together,

7 these results underscore the importance of developing the RAP dose conversion method

8 for nonclinical and clinical future studies.

\section{Conclusions}

The RAP dose conversion method renovated by the new formalism was able to

12 estimate the $\left[{ }^{211} \mathrm{At}\right] \mathrm{MABG}$ absorbed dose using the pharmacokinetics of $\left[{ }^{131} \mathrm{I}\right] \mathrm{MIBG}$

13 through the use of a behavior map of an optimal timing of a single biodistribution

14 measurement. The present formalism revealed optimizing imaging time points on

15 absorbed dose conversion between two radiopharmaceuticals. Further analysis and

16 clinical data will be needed to elucidate the validity of a behavior map of an optimal

17 timing of a single measurement for TAT. 


\section{Abbreviations}

2 HL: Half-life;

$3 \quad \% \mathrm{ID} / \mathrm{g}$ : Percent injected dose per gram;

4 RAP: RAtio of Pharmacokinetics;

5 TIAC: Time integration activity concentration;

6 MIRD: Medical internal radiation dose

7

8 Acknowledgements

$9 \quad$ Not applicable.

\section{Authors' contributions}

12 Conceptualization: TS; Data validation and curation: SW, HH, YO, IS; Experimental

13 design: TS, YI, NU, TH, YT; Modeling and analysis: TS, SM; Scientific writing: TS, SM,

$14 \mathrm{HH}, \mathrm{TH}$; Securance of funding: NSI. All authors read and approved the final manuscript.

\section{Funding}

17 This work was supported in part by a KAKENHI grant (JP19H04296) from the Japan

18 Society for the Promotion of Science to Dr. N. Suzui. 
$2 \quad$ Availability of data and materials

3 The datasets used and/or analyzed in the present study are available from the

4 corresponding author on reasonable request.

5

6 Declarations

$7 \quad$ Ethics approval and consent to participate

8 This article does not contain any studies with animals and human participants performed

9 by any of the authors.

10

11 Consent for publication

12 Not applicable.

13

14 Competing interests

15 The authors declare that they have no competing interests.

$17 \quad$ Author details

$18{ }^{1}$ Quantum Beam Science Research Directorate, National Institutes for Quantum and 
1 Radiological Science and Technology, 1233 Watanuki-machi, Takasaki 370-1292, Japan

$2 \quad{ }^{2}$ Department of Bioimaging Information Analysis, Gunma University Graduate School of

3 Medicine, 3-39-22 Showa, Maebashi 371-8511, Japan

$4 \quad{ }^{3}$ Department of Molecular Imaging and Theranostics, National Institutes for Quantum and

5 Radiological Science and Technology, 4-9-1 Anagawa, Inage-ku, Chiba 263-8555, Japan

$6{ }^{4}$ Advanced Clinical Research Center, Fukushima Medical University, 1 Hikariga-oka,

7 Fukushima 960-1295, Japan

$8{ }^{5}$ Department of Diagnostic Radiology and Nuclear Medicine, Gunma University

9 Graduate School of Medicine, 3-39-22 Showa, Maebashi 371-8511, Japan

\section{$12 \quad$ References}

13 1. van Hulsteijn LT, Niemeijer ND, Dekkers OM, Corssmit EP. ${ }^{131}$ I-MIBG therapy for

14 malignant paraganglioma and phaeochromocytoma: systematic review and meta-analysis.

15 Clin Endocrinol. 2014;80:487-501.

16 2. Roncali E, Capala J, Benedict SH, Akabani G, Bednarz B, Bhadrasain V, Bolch WE,

17 Buchsbaum J, Clarke BN, Coleman N, Dewaraja YK, Frey EC, Ghaly M, Grudzinski J,

18 Hobbs RF, Howell RW, Humm JL, Kunos C, Larson SM, Lin FI, Madsen MT, Mirzadeh 
1 S, Morse DL, Pryma DA, Sgouros G, James SS, Wahl RL, Xiao Y, Zanzonico PB,

2 Zukotynski K. Overview of the First NRG-NCI Workshop on Dosimetry of Systemic

3 Radiopharmaceutical Therapy (RPT). J Nucl Med. 2020; doi:

$4 \quad 10.2967 /$ jnumed.120.255547.

5 3. Madsen MT, Menda Y, O’Dorisio TM, O’Dorisio MS. Technical Note: Single time

6 point dose estimate for exponential clearance. Med Phys. 2018; 45: 2318-24.

7 4. Sakashita T, Watanabe S, Hanaoka H, Ohshima Y, Ikoma Y, Ukon N, Sasaki I, Higashi

8 T, Higuchi T, Tsushima Y, Ishioka SN. Absorbed dose simulation of meta $^{2}{ }^{211}$ At-astato-

9 benzylguanidine using pharmacokinetics of ${ }^{131}$ I-MIBG and a novel dose conversion

10 method, RAP. Ann Nucl Med. 2021;35:121-31.

11 5. Vaidyanathan G, Friedman HS, Keir ST, Zalutsky MR. Evaluation of meta$12\left[{ }^{211} \mathrm{At}\right]$ astatobenzylguanidine in an athymic mouse human neuroblastoma xenograft 13 model. Nucl Med Biol. 1996;23:851-6.

14 6. Ohshima Y, Sudo H, Watanabe S, Nagatsu K, Tsuji AB, Sakashita T, Ito YM, Yoshinaga

$15 \mathrm{~K}$, Higashi T, Ishioka NS. Antitumor effects of radionuclide treatment using $\alpha$-emitting 16 meta $^{211}$ At-astato-benzylguanidine in a PC12 pheochromocytoma model. Eur J Nucl Med 17 Mol Imaging. 2018;45:999-1010.

18 7. Watanabe S, Hanaoka H, Liang JX, Iida Y, Endo K, Ishioka NS. PET imaging of 
1 norepinephrine transporter-expressing tumors using ${ }^{76} \mathrm{Br}$-meta-bromobenzylguanidine. $\mathrm{J}$

$2 \quad$ Nucl Med. 2010;51:1472-9.

3 8. Spetz J, Rudqvist N, Forssell-Aronsson E. Biodistribution and dosimetry of free ${ }^{211} \mathrm{At}$,

$4 \quad{ }^{125} \mathrm{I}-$ and ${ }^{131} \mathrm{I}-$ in rats. Cancer Biother Radiopharm. 2013;28:657-64.

5 9. Christoph AU, Martina B, Raffaella MS, Andreas T, Roger S, Nicholas PM, Cristina

6 M. ${ }^{44}$ Sc-PSMA-617 for radiotheragnostics in tandem with ${ }^{177}$ Lu-PSMA-617-preclinical

7 investigations in comparison with ${ }^{68} \mathrm{Ga}-\mathrm{PSMA}-11$ and ${ }^{68} \mathrm{Ga}-\mathrm{PSMA}-617$. EJNMMI Res.

$8 \quad 2017 ; 7(1): 9$.

9 10. Stabin MG, Sparks RB, Crowe E. OLINDA/EXM: the second-generation personal 10 computer software for internal dose assessment in nuclear medicine. J Nucl Med. $11 \quad 2005 ; 46: 1023-7$.

12 11. Hjornevik T, Martinsen AC, Hagve SE, Andersen MW, Mørk AC, Fjeld JG, Ruud E.

13 Experiences from introducing standardized high dose ${ }^{131} \mathrm{I}-\mathrm{mIBG}$ treatment of children

14 with refractory neuroblastoma: differences in effective dose to patients and exposure to 15 caregivers. J Nucl Med Radiat Ther. 2015;6:6. 


\section{$1 \quad$ Figure legends}

2 Fig. 1 Comparison between converted absorbed doses of $\left[{ }^{131} \mathrm{I}\right] \mathrm{MIBG}[5],\left[{ }^{211} \mathrm{At}\right] \mathrm{MABG}$

3 [6], $\left[{ }^{125} \mathrm{I}\right] \mathrm{MIBG}$ [7], and $\left[{ }^{77} \mathrm{Br}\right] \mathrm{MBBG}$ [7] and the true target absorbed dose of

$4 \quad\left[{ }^{211} \mathrm{At}\right] \mathrm{MABG}[5]$. (a) Converted absorbed doses from [ $\left.{ }^{131} \mathrm{I}\right] \mathrm{MIBG}$ [5] as a reference, and

5 absorbed dose of $\left[{ }^{211} \mathrm{At}\right] \mathrm{MABG}[5]$ as a true target. [ $\left.{ }^{131} \mathrm{I}\right] \mathrm{MIBG}[5]$ indicates the absorbed

6 dose of an organ or tumor tissue when alpha rays derived from ${ }^{211}$ At were emitted by the

7 number of radioactive decays of ${ }^{131} \mathrm{I}$. "with half-life (HL)" shows a converted absorbed

8 dose of $\left[{ }^{131} \mathrm{I}\right] \mathrm{MIBG}[5]$ corrected by the physical half-life of ${ }^{131} \mathrm{I}$ and radioactively

9 decayed with the physical half-life of ${ }^{211}$ At, indicating the physical part of equation (5).

10 "with HL + RAtio of Pharmacokinetics (RAP)" is an absorbed dose of "with HL"

11 converted using the representative RAP coefficient. (b) Same as (a) except that $\left[{ }^{211} \mathrm{At}\right]$

12 MABG [6] is a reference. (c) Same as (a) except that $\left[{ }^{125} \mathrm{I}\right] \mathrm{MIBG}[7]$ is a reference. (d)

13 Same as (a) except that $[77 \mathrm{Br}] \mathrm{MBBG}[7]$ is a reference.

15 Fig. 2 Inverse of the RAP coefficient at $t$ (solid line) and the representative RAP 16 coefficient (square) on [ $\left.{ }^{131} \mathrm{I}\right] \mathrm{MIBG}$ [5]. (a) Heart, liver, kidney, and intestine. (b) Blood, 17 adrenals and stomach, and tumor tissue. 
1 Fig. 3 Behavior maps of an optimal timing (solid line) on ${ }^{211}$ At-labeled target compounds.

$2 T_{p A 1}$ and $T_{b A 1}$ are the physical HL and HL of the biological clearance of a target,

3 respectively. $T_{b A 2}$ is the half-life of the biological clearance of a reference. Behavior maps

4 of $T_{b A 2}$ in these cases of 5, 10, 15, 20, 25, and $50 \mathrm{~h}$ are presented in the panels of (a), (b),

5 (c), (d), (e), and (f), respectively. The dashed and dotted lines represent optimal timing in

6 the cases of 0.95 and 1.05 , and 0.9 and 1.1 times converted absorbed doses, respectively.

8 Fig. 4 Behavior maps of optimal timing (solid line) on radiolabeled target compounds

9 with a physical HL of $10 \mathrm{~d} . T_{p A 1}$ and $T_{b A 1}$ are the physical HL and HL of the biological

10 clearance of a target, respectively. $T_{b A 2}$ is the half-life of the biological clearance of a

11 reference. Behavior maps of $T_{b A 2}$ in these cases of $25,50,100$, and $200 \mathrm{~h}$ are presented in

12 the panels of (a), (b), (c), and (d), respectively. The dashed and dotted lines represent

13 optimal timing in the cases of 0.95 and 1.05 , and 0.9 and 1.1 times converted absorbed

14 doses, respectively.

16 Fig. 5 Behavior maps of an optimal timing (solid line) on radiolabeled target compounds

17 with a physical HL of $10 \mathrm{~d}$. $T_{p A 1}$ and $T_{b A 1}$ are the physical HL and HL of the biological

18 clearance of a target, respectively. (a) $\mathrm{HL}$ of the biological clearance of a reference, $T_{b A 2}$,

19 of $25 \mathrm{~h}$. (b) HL of the biological clearance of a reference, $T_{b A 2}$, of $1 \mathrm{~h}$. The dashed and 
1 dotted lines represent optimal timing in the cases of 0.95 and 1.05 , and 0.9 and 1.1 times

2 converted absorbed doses, respectively.

3

4 Fig. 6 Example of a RAP dose conversion using an optimal timing behavior map for a

5 single biodistribution measurement (\%ID/g) on ${ }^{211}$ At-labeled target compounds. First, (a)

6 plotted values converted to logarithms of $\left[{ }^{131} \mathrm{I}\right] \mathrm{MIBG}$ [5] corrected by the physical half-

7 life of ${ }^{131}$ I, i.e., a biological component, and fitted by the linear function (the dashed line).

8 Second, (b) draws the behavior map of an optimal timing (solid line) on a target

9 compound with a physical HL of $7.2 \mathrm{~h}$ and a reference with a $6.9 \mathrm{~h}$ HL of biological

10 clearance, $T_{b A 2}$, which was derived from the fitting curve on panel (a). The dashed and

11 dotted lines represent optimal timing in the cases of 0.95 and 1.05 , and 0.9 and 1.1 times

12 converted absorbed doses, respectively. Finally, (c) converted absorbed doses of

13 [ ${ }^{131}$ I]MIBG [5], "with HL", "with HL + RAP", and a true target absorbed dose of

$14 \quad\left[{ }^{211} \mathrm{At}\right] \mathrm{MABG}[5]$. 

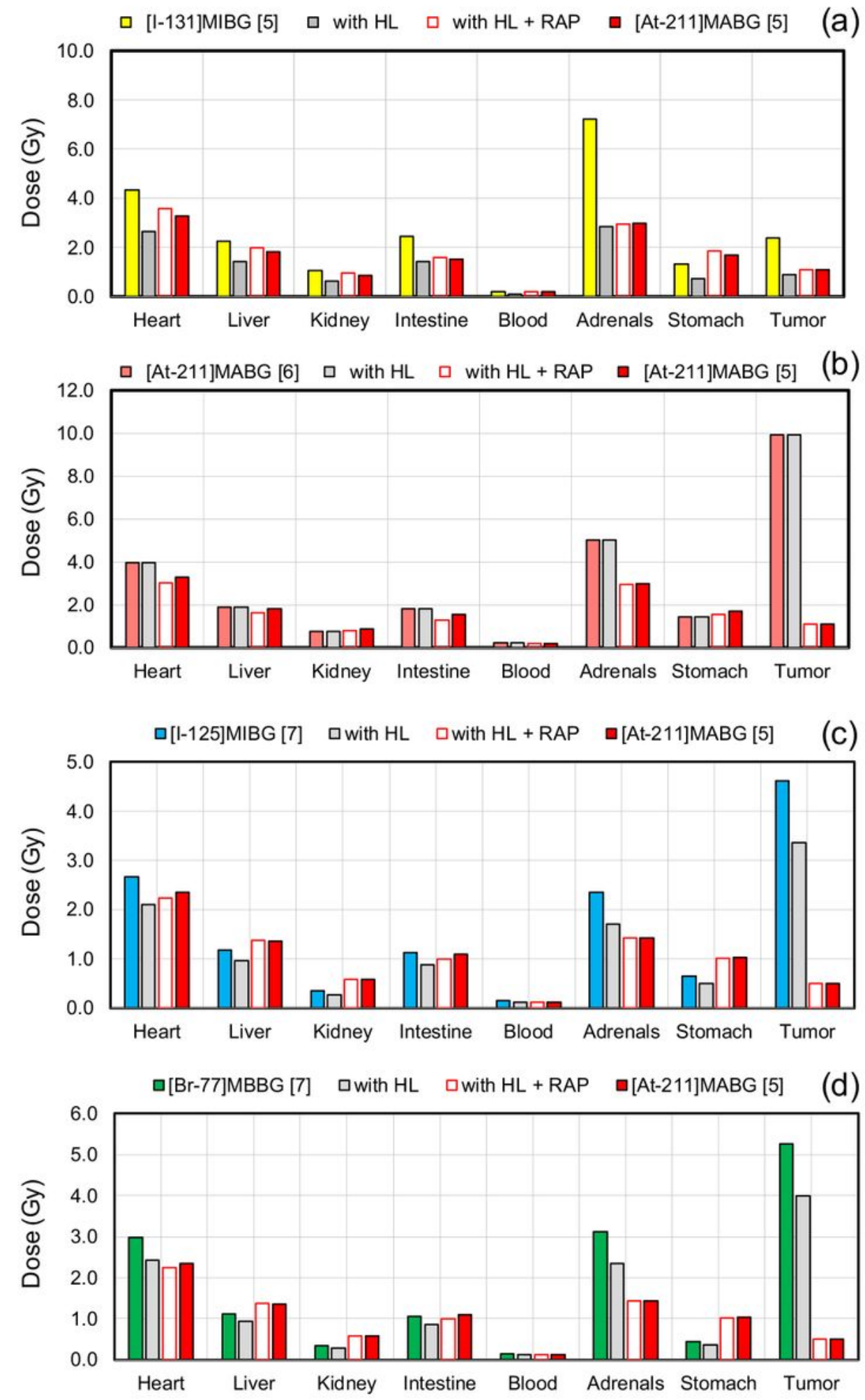

\section{Figure 1}

Comparison between converted absorbed doses of [1311]MIBG [5], [211At]MABG [6], [125I]MIBG [7], and [77Br]MBBG [7] and the true target absorbed dose of [211At]MABG [5]. (a) Converted absorbed doses from [131I]MIBG [5] as a reference, and absorbed dose of [211At]MABG [5] as a true target. [131I]MIBG [5] 
indicates the absorbed dose of an organ or tumor tissue when alpha rays derived from 211At were emitted by the number of radioactive decays of 131I. "with half-life $(\mathrm{HL})$ " shows a converted absorbed dose of [131I]MIBG [5] corrected by the physical half-life of $131 \mathrm{I}$ and radioactively decayed with the physical half-life of 211At, indicating the physical part of equation (5)."with HL + RAtio of

Pharmacokinetics (RAP)" is an absorbed dose of "with HL" converted using the representative RAP coefficient. (b) Same as (a) except that [211At] MABG [6] is a reference. (c) Same as (a) except that [125I]MIBG [7] is a reference. (d) Same as (a) except that [77Br]MBBG [7] is a reference.

(a)
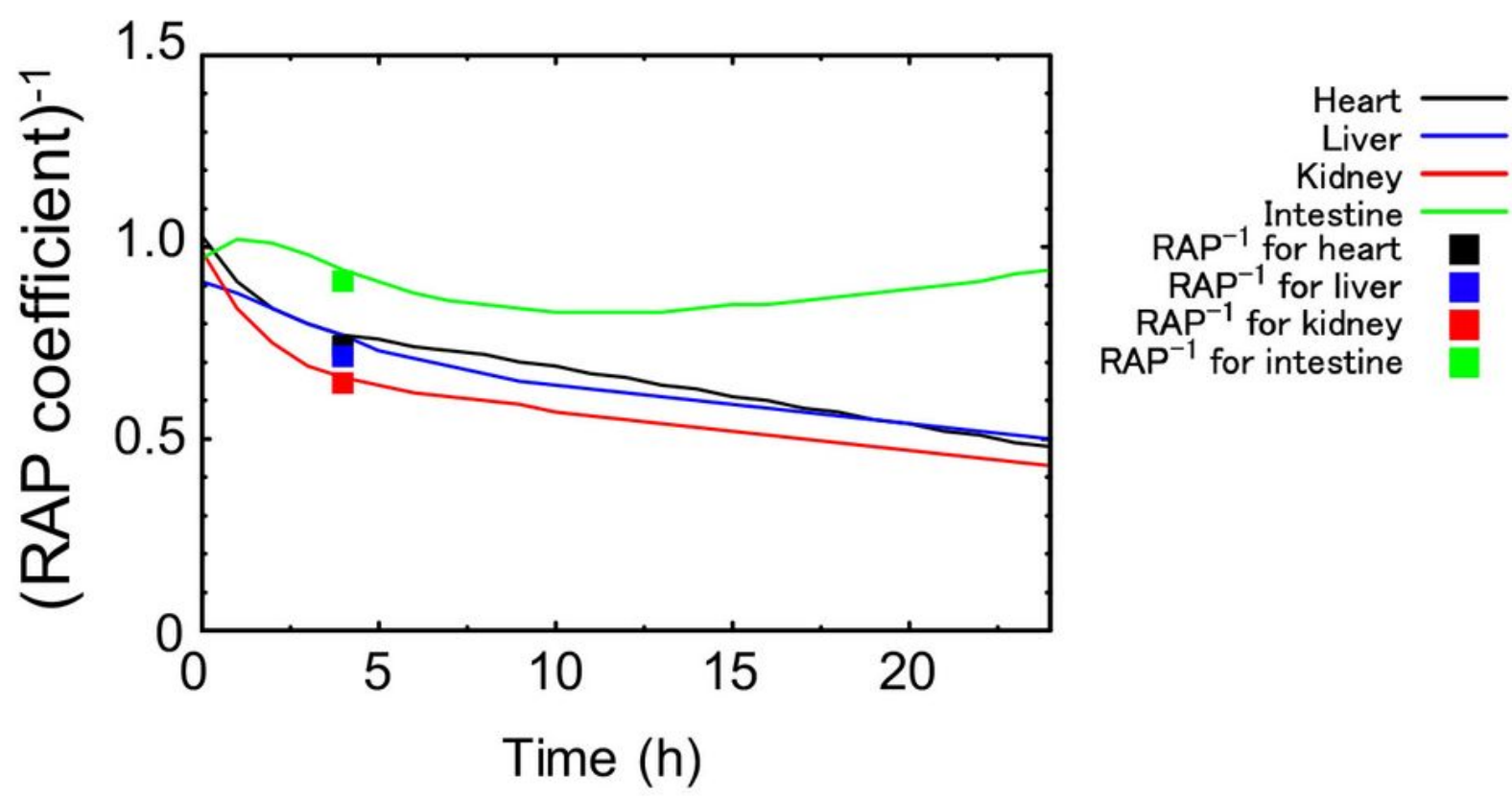

(b)
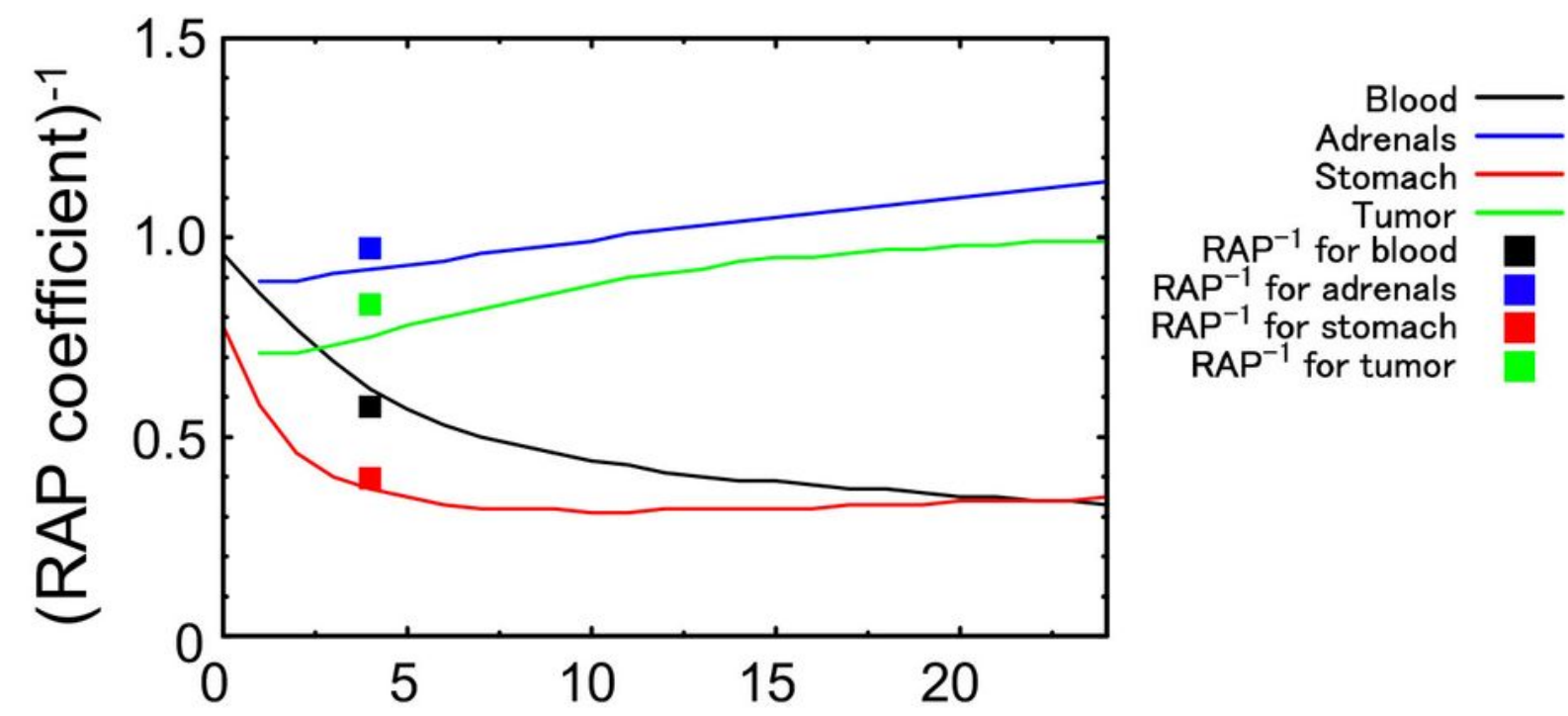

Time $(h)$ 
Figure 2

Inverse of the RAP coefficient at $t$ (solid line) and the representative RAP coefficient (square) on [1311]MIBG [5]. (a) Heart, liver, kidney, and intestine. (b) Blood, adrenals and stomach, and tumor tissue.

(a)

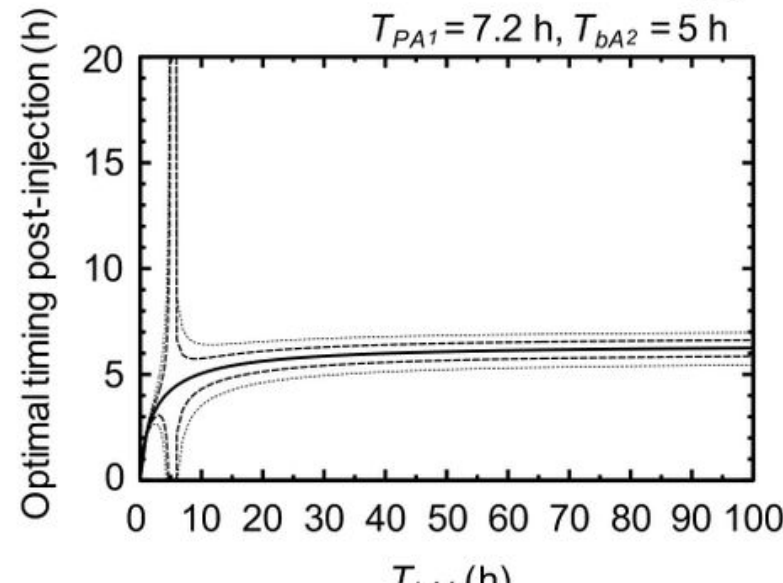

(c)
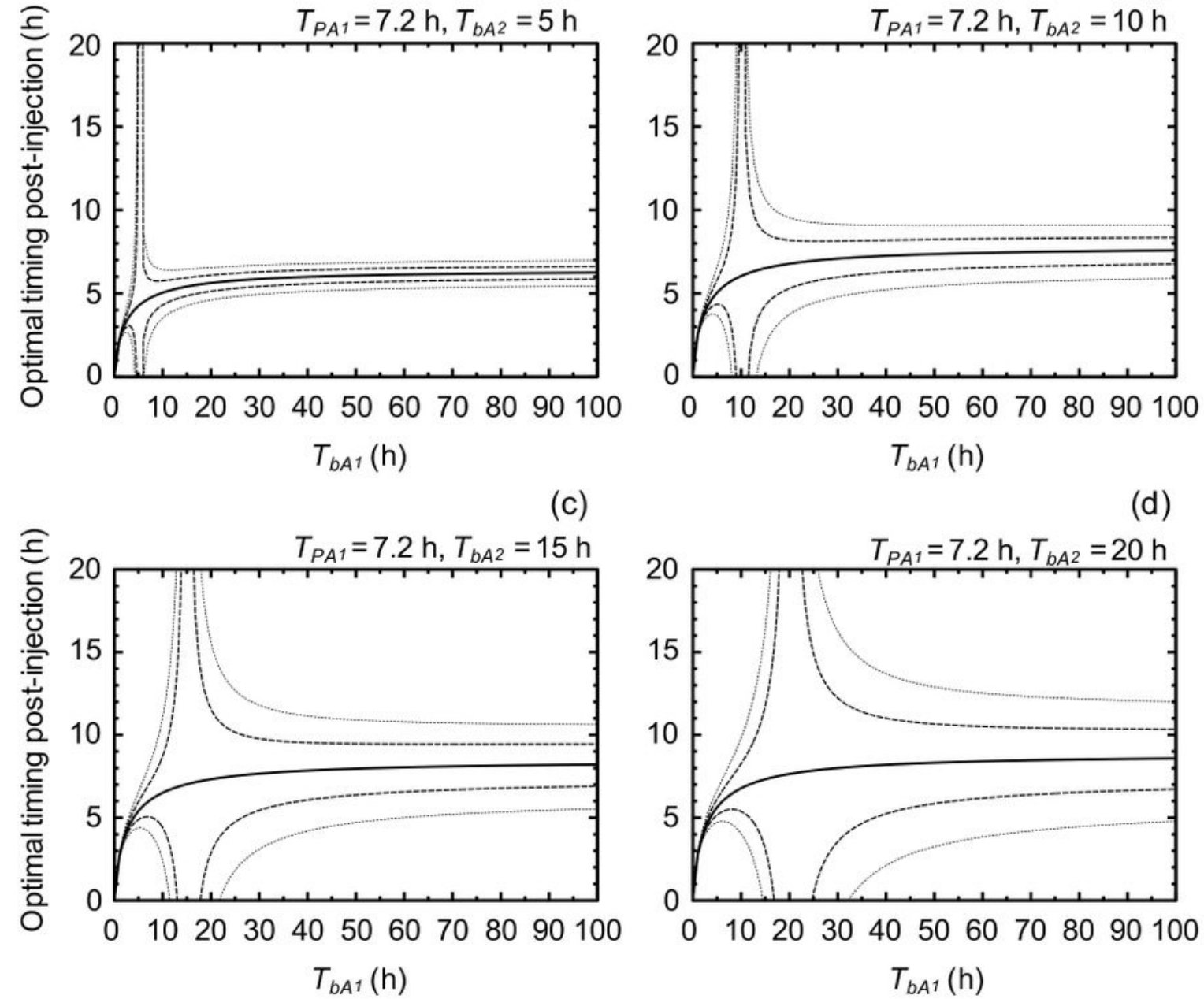

)

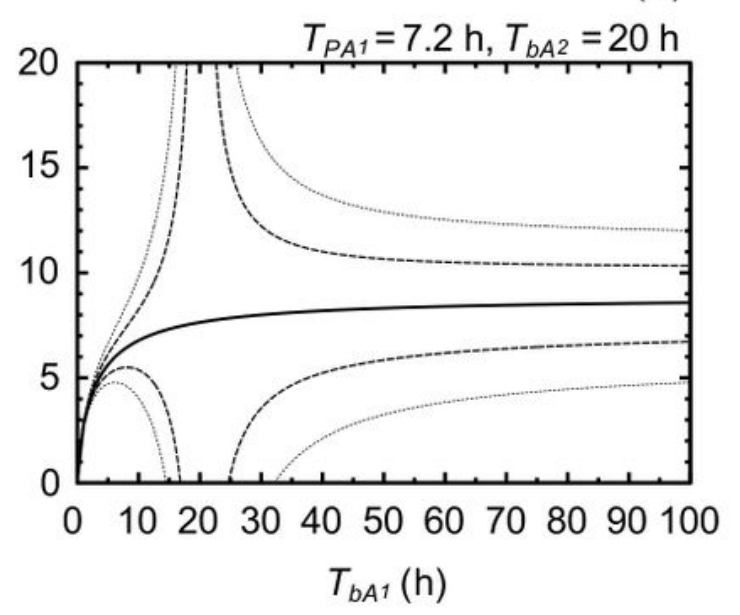

(e)

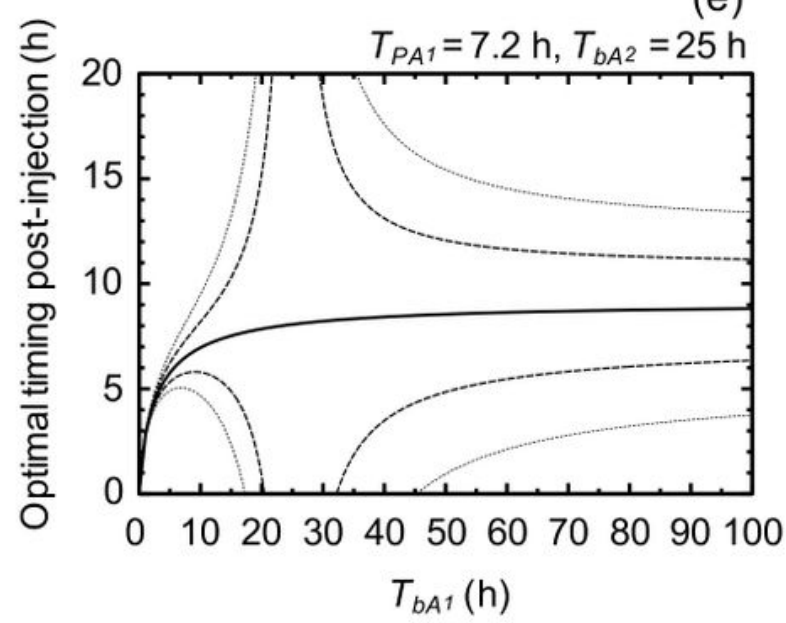

(f)

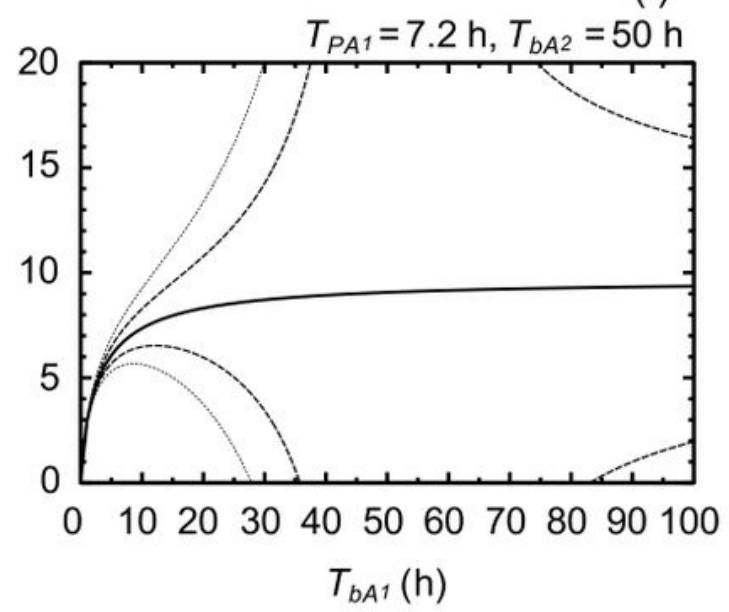

Figure 3 
Behavior maps of an optimal timing (solid line) on 211At-labeled target compounds. TpA1 and TbA1 are the physical $\mathrm{HL}$ and $\mathrm{HL}$ of the biological clearance of a target, respectively. TbA2 is the half-life of the biological clearance of a reference. Behavior maps of TbA2 in these cases of $5,10,15,20,25$, and $50 \mathrm{~h}$ are presented in the panels of (a), (b), (c), (d), (e), and (f), respectively. The dashed and dotted lines represent optimal timing in the cases of 0.95 and 1.05 , and 0.9 and 1.1 times converted absorbed doses, respectively.

(a)

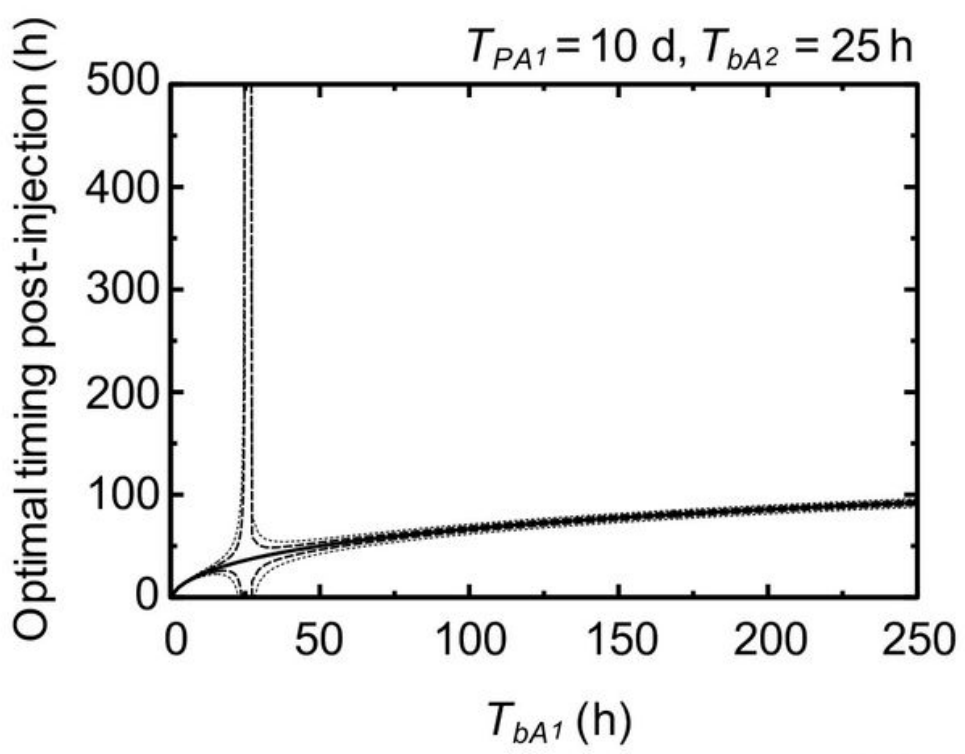

(c)

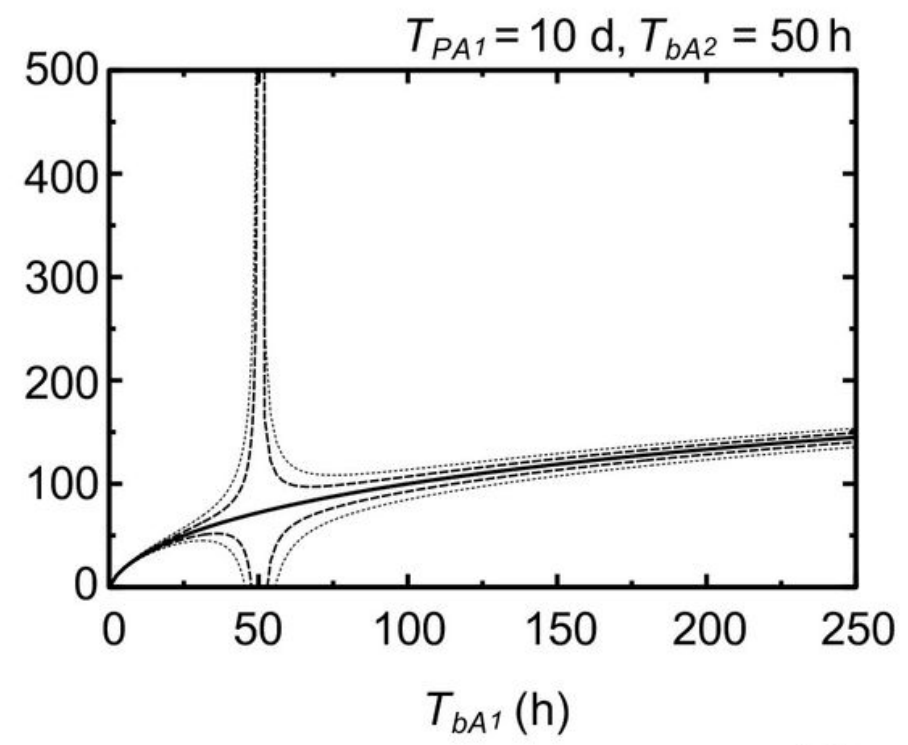

(d)
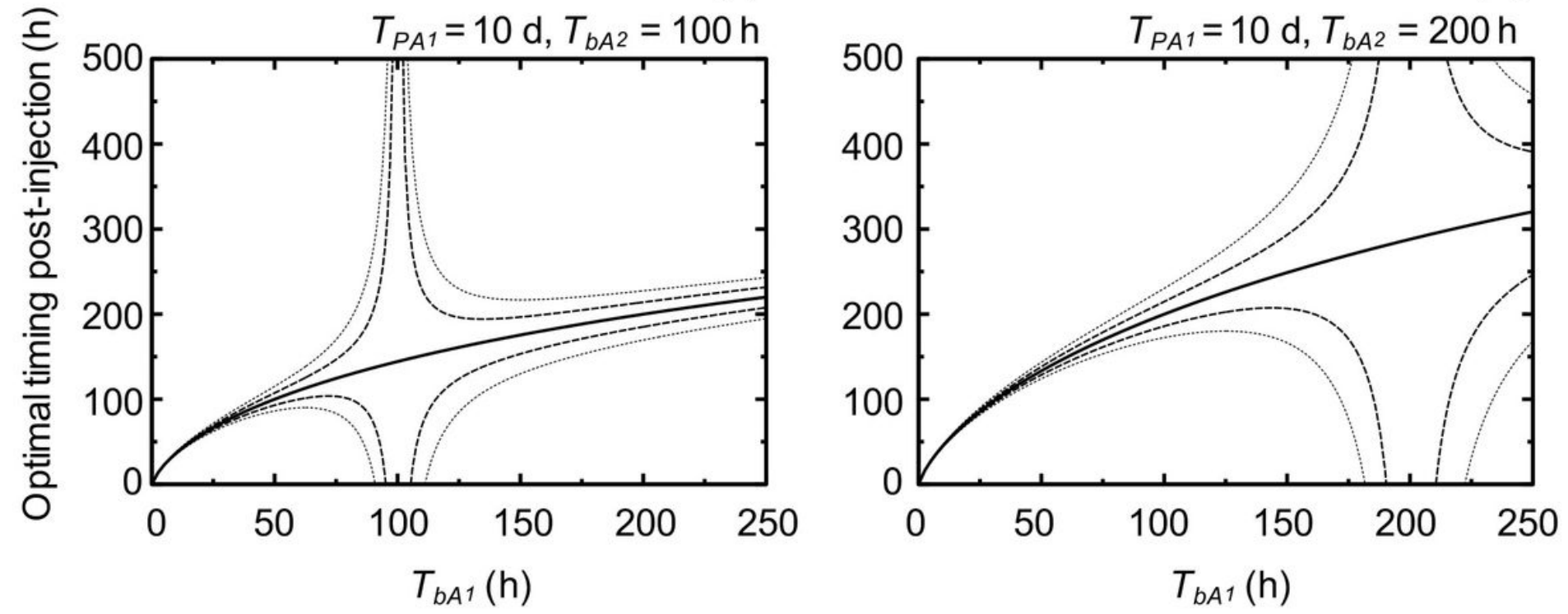

Figure 4

Behavior maps of optimal timing (solid line) on radiolabeled target compounds with a physical HL of 10 d. TpA1 and TbA1 are the physical $\mathrm{HL}$ and $\mathrm{HL}$ of the biological clearance of a target, respectively. TbA2 is the half-life of the biological clearance of a reference. Behavior maps of TbA2 in these cases of 25, 50, 100 , and $200 \mathrm{~h}$ are presented in the panels of (a), (b), (c), and (d), respectively. The dashed and dotted 
lines represent optimal timing in the cases of 0.95 and 1.05 , and 0.9 and 1.1 times converted absorbed doses, respectively.

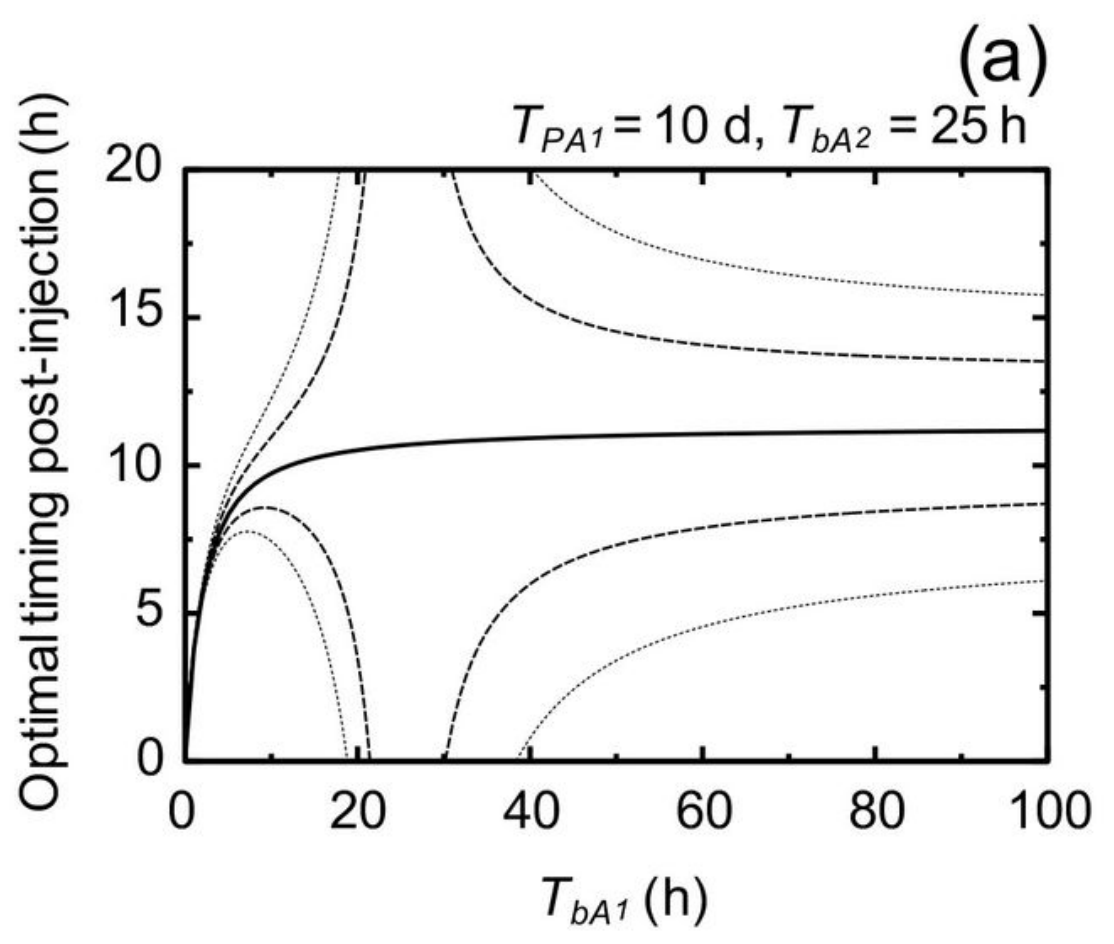

(b)

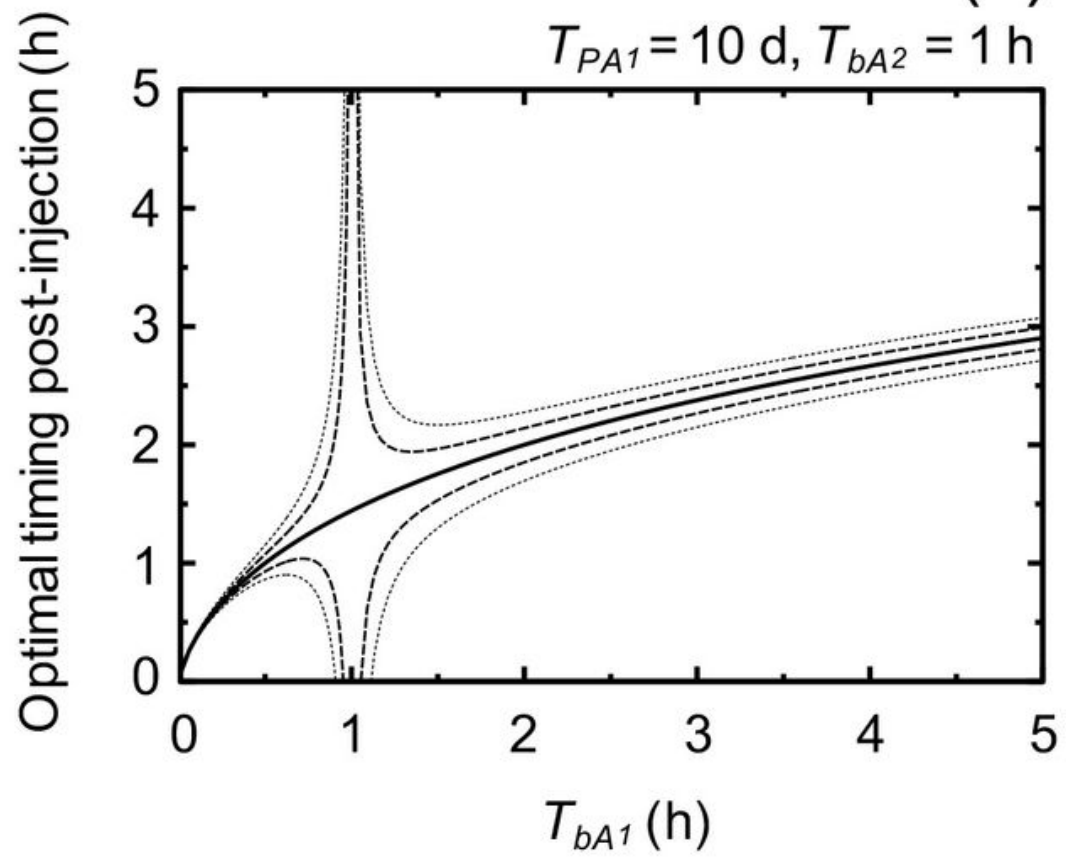

Figure 5

Behavior maps of an optimal timing (solid line) on radiolabeled target compounds with a physical HL of $10 \mathrm{~d}$. TpA1 and TbA1 are the physical HL and HL of the biological clearance of a target, respectively. (a) $\mathrm{HL}$ of the biological clearance of a reference, TbA2, of $25 \mathrm{~h}$. (b) $\mathrm{HL}$ of the biological clearance of a 
reference, TbA2, of $1 \mathrm{~h}$. The dashed and dotted lines represent optimal timing in the cases of 0.95 and 1.05 , and 0.9 and 1.1 times converted absorbed doses, respectively.

(a)

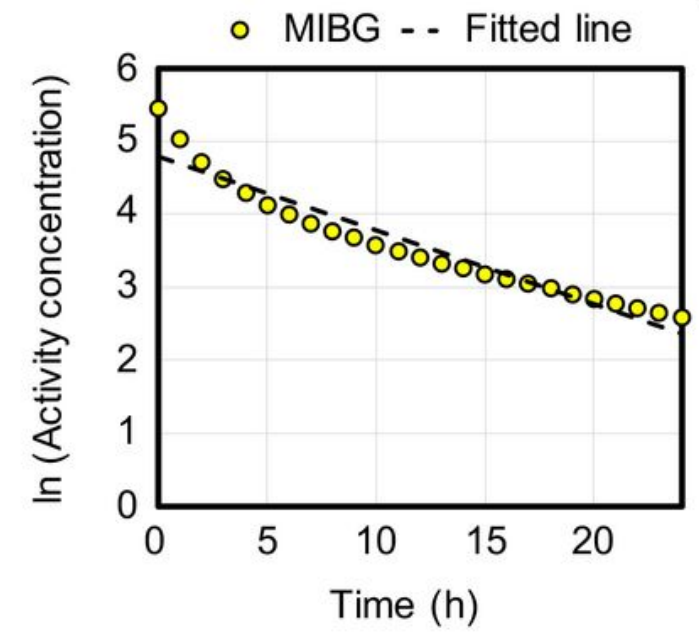

(b)

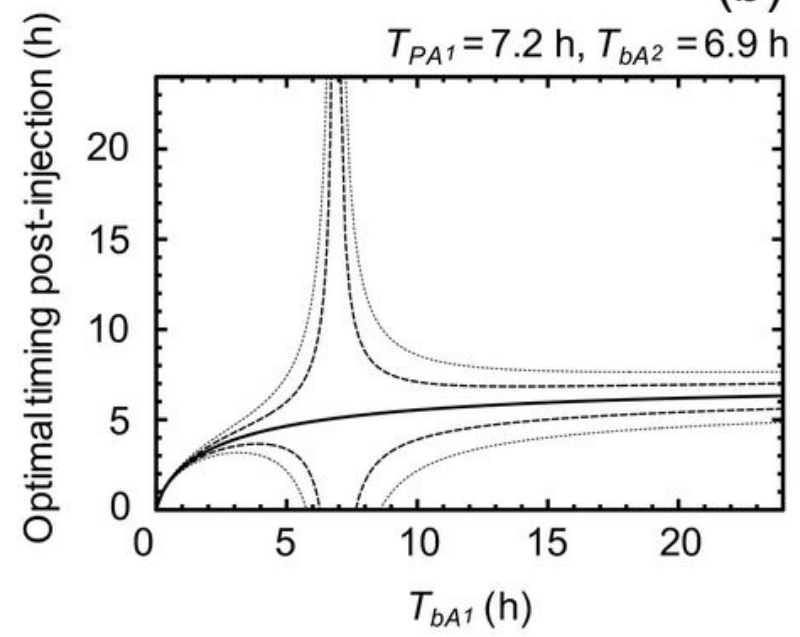

(c)

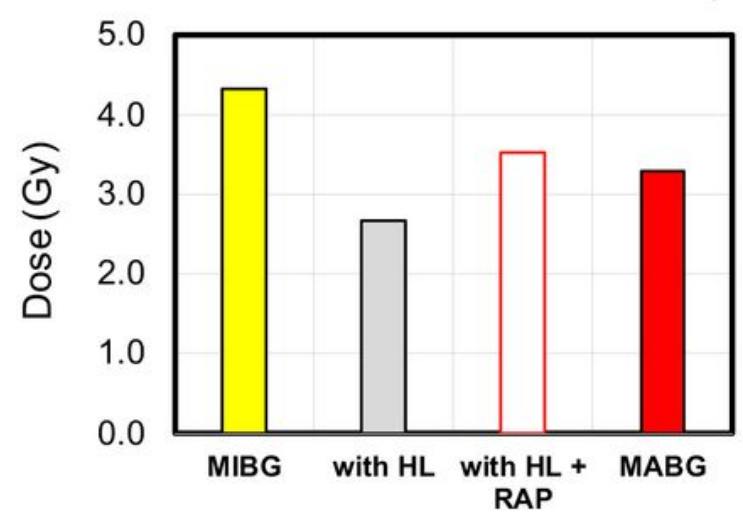

Figure 6

Example of a RAP dose conversion using an optimal timing behavior map for a single biodistribution measurement (\%ID/g) on 211 At-labeled target compounds. First, (a) plotted values converted to logarithms of [131]]MIBG [5] corrected by the physical half-life of 131।, i.e., a biological component, and 
fitted by the linear function (the dashed line). Second, (b) draws the behavior map of an optimal timing (solid line) on a target compound with a physical $\mathrm{HL}$ of $7.2 \mathrm{~h}$ and a reference with a $6.9 \mathrm{~h} \mathrm{HL}$ of biological clearance, TbA2, which was derived from the fitting curve on panel (a). The dashed and dotted lines represent optimal timing in the cases of 0.95 and 1.05, and 0.9 and 1.1 times converted absorbed doses, respectively. Finally, (c) converted absorbed doses of [131I]MIBG [5], "with HL", "with HL + RAP", and a true target absorbed dose of [211At]MABG [5]. 